Supporting Information

\title{
Synthesis and Characterization of Linear Poly(dialkylstannane)s
}

\author{
Fabien Choffat, ${ }^{\star}$ Susanne Käser, ${ }^{*}$ Pascal Wolfer ${ }^{\star}$ Daniel Schmid, ${ }^{\ddagger}$ Raffaele Mezzenga,${ }^{\S, \dagger}$ \\ Paul Smith ${ }^{\ddagger}$ and Walter Caseri**;
}

Department of Materials, ETH Zürich, Wolfgang-Pauli-Strasse 10, 8093 Zürich, Switzerland,

Department of Physics and Fribourg Center for Nanomaterials, University of Fribourg, Ch. du Musée 3, CH-1700 Fribourg, Switzerland,

Nestlé Research Center, Vers-Chez-les-Blancs, CH-1000 Lausanne 26, Switzerland. 


\section{Experimental}

1.1. Synthesis of tetraalkylstannanes $\left(\mathbf{R}_{\mathbf{4}} \mathbf{S n}\right)$. Tetrachlorostannane (quantities see Table 1a) was dissolved in hexane. The hexane solution was added slowly (typically over a period of $1 \mathrm{~h}$ ) to ice-cooled alkyl magnesium halide (bromide or chloride) / diethyl ether solution with a dropping funnel. The reaction vessel was connected to a balloon filled with argon in order to protect the substances from oxygen and moisture. After the addition of the tetrachlorostannane solution was completed, the reaction mixture was boiled under reflux for $2 \mathrm{~h}$. Thereafter the diethyl ether was removed by distillation under argon at ambient pressure, followed by addition of toluene (the same volume as the volume of removed diethyl ether). The replacement of diethyl ether by toluene allowed an increase in the reaction temperature to ca. $90{ }^{\circ} \mathrm{C}$, which is known to increase the yields of the conversion reactions with Grignard's reagent, ${ }^{1}$ the reaction mixture was kept for $1 \mathrm{~h}$ under reflux. ${ }^{2,3}$ Thereafter the mixture was cooled with ice water, and a solution composed of $5 \% \mathrm{~m} / \mathrm{m}$ concentrated $\mathrm{HCl}$ and $95 \% \mathrm{~m} / \mathrm{m}$ water was slowly added until the aqueous phase became clear. The organic phase, which separated from the aqueous phase, was removed with a separating funnel, dried with anhydrous magnesium sulfate and filtered through celite. The solvents were removed with a rotary evaporator at a bath temperature of $40^{\circ} \mathrm{C}$, initially at $335 \mathrm{mbar}$ for removal of hexane and in a second step commencing at 77 mbar and finally down to 25 mbar for the elimination of the toluene. The resulting product was then dried in the vacuum (ca. 0.5 mbar) overnight. No further purification was performed, if not otherwise indicated. The tetraalkylstannes thus obtained were analyzed by NMR spectroscopy (Table 2, Table 3) and DSC for determination of their melting temperatures (Table 1a; melting temperatures of tetraalkylstannanes which solidified below $-65^{\circ} \mathrm{C}$ could not be detected). 


\subsection{Synthesis of dialkyldichlorostannanes $\left(\mathbf{R}_{2} \mathbf{S n C l}_{2}\right)$. Dialkyldichlorostannanes were}

obtained by the Kocheshkov reaction of tetraalkylstannanes with $\mathrm{SnCl}_{4}{ }^{4,5}$ Neat tetraalkylstannane was poured into a two-necked round-bottom flask equipped with reflux condenser (all quantities see Table 1b). The top of the condenser was connected with a balloon filled with argon in order to protect the reaction mixture from oxygen and moisture. The second neck was closed by a septum through which neat tetrachlorostannane was added with a syringe. Thereafter the reaction mixture was heated to $100^{\circ} \mathrm{C}$ for $2 \mathrm{~h}$ and to $220^{\circ} \mathrm{C}$ for an additional $4 \mathrm{~h}$. The resulting product was recrystallized from pentane ${ }^{6}$ (ca. $100 \mathrm{ml} / 20 \mathrm{~g}$ ) and dried in vacuum (ca. 0.5 mbar) for $24 \mathrm{~h}$. The compounds were obtained in the from of colorless crystals and were analyzed by NMR spectroscopy (Table 2, Table 3), elemental analysis (Table 4) and DSC for the determination of the melting temperature (Table 1b).

1.3. Synthesis of dialkylstannanes $\left(\mathbf{R}_{2} \mathrm{SnH}_{2}\right)$. Dialkyldichlorostannanes (quantities see Table 1c) were reduced with an excess of $\mathrm{Li}\left[\mathrm{AlH}_{4}\right]$ by the method developed by Finholt, Bond, Wilzbach and Schlesinger ${ }^{7,8}$ as described in detail previously. ${ }^{2,6}$ A solution of dialkyldichlorostannane in diethyl ether (typically $0.4 \mathrm{M}$ ) was added dropwise with a dropping funnel to the same volume of ice-cooled $\mathrm{Li}\left[\mathrm{AlH}_{4}\right]$ suspension in diethyl ether, which was protected from air by argon counter flow. After addition during approximately $1 \mathrm{~h}$, the reaction mixture was stirred for an additional hour at $0{ }^{\circ} \mathrm{C}$, before the ice bath was removed in order to allow the mixture to warm up to room temperature. The reaction mixture was then quenched with ice water and the organic phase was separated from the water phase with a separating funnel. The ether phase was dried over anhydrous magnesium sulfate. The solution was filtered through celite and the solvent was removed with a rotary evaporator at $40{ }^{\circ} \mathrm{C}$ and ambient pressure. The resulting liquids were distilled or recrystallized at low temperatures (cf. Table 1c) depending on their boiling temperature. Thereafter the liquids were collected in 
containers which were closed with a septum and degassed by bubbling argon by a syringe needle which was immersed in the dialkylstannane for at least $5 \mathrm{~min}$ (this procedure was found to be essential). The containers were immediately completely wrapped with soft tissue and aluminum foil to protect them from light and stored in a refrigerator at $-20{ }^{\circ} \mathrm{C}$ (N.B. a sample of $\mathrm{Bu}_{2} \mathrm{SnH}_{2}$ which was stored under these conditions for 13 months yielded a polystannane with the same characteristics as a polymer obtained from a freshly prepared $\mathrm{Bu}_{2} \mathrm{SnH}_{2}$ ). The obtained dialkylstannanes were analyzed by NMR spectroscopy (Table 2 , Table 3), infrared spectroscopy (Table 1c) and elemental analysis (Table 4). Since the dialkylstannanes are sensitive to the ambient, they were analyzed rapidly after synthesis. The difference between experimental and calculated carbon content of $0.59 \%$ of $\operatorname{Dod}_{2} \mathrm{SnH}_{2}$ might be due the presence of some solvent. 
Table 1: Synthesis parameters for monomer precursors $\mathrm{R}_{4} \mathrm{Sn}(\mathrm{a}), \mathrm{R}_{2} \mathrm{SnCl}_{2}$ (b) and $\mathrm{R}_{2} \mathrm{SnH}_{2}$.

Also included are selected analytical data of monomer precursors. ( $T_{\mathrm{m}}$ : melting temperature at ambient pressure, $v(\mathrm{Sn}-\mathrm{H})$ : IR-absorbtion vibration of the $\mathrm{Sn}-\mathrm{H}$ bond, $T_{\mathrm{b}}$ : boiling temperature at the indicated pressure $p_{\mathrm{b}}$.

(a)

\begin{tabular}{|c|c|c|c|c|c|c|c|c|}
\hline \multirow{2}{*}{$\mathbf{R}$} & \multirow{2}{*}{$\underset{[\mathrm{g}(\mathbf{m m o l})]}{\mathrm{SnCl}_{4}}$} & \multirow{2}{*}{$\begin{array}{c}\text { Hexane } \\
{[\mathrm{ml}]}\end{array}$} & \multicolumn{3}{|c|}{$\begin{array}{c}\text { Alkylmagnesium halide } \mathrm{RMgX} \text { : } \\
\text { volume } V \text { and quantity } n\end{array}$} & \multirow{2}{*}{$\begin{array}{c}\mathbf{R}_{4} \mathbf{S n} \\
{[\mathrm{g}(\mathbf{m m o l})]}\end{array}$} & \multirow{2}{*}{$\begin{array}{l}\text { Yield } \\
{[\%]}\end{array}$} & \multirow{2}{*}{$\begin{array}{c}\boldsymbol{T}_{\mathbf{m}} \\
{\left[{ }^{\circ} \mathbf{C}\right]}\end{array}$} \\
\hline & & & $\mathbf{V}[\mathrm{ml}]$ & $\mathbf{n}[\mathrm{mmol}]$ & $\mathbf{X}$ & & & \\
\hline Pr & $10.42(40)$ & 100 & 100 & 200 & $\mathrm{Br}$ & $11.28(39)$ & 97 & $<-50$ \\
\hline Pe & $10.42(40)$ & 100 & 100 & 200 & $\mathrm{Cl}$ & $15.34(38)$ & 95 & $<-50$ \\
\hline Hex & $11.27(43)$ & 100 & 100 & 200 & $\mathrm{Br}$ & $19.57(40)$ & 93 & $<-50$ \\
\hline Dod & $41.7(160)$ & 400 & 800 & 800 & $\mathrm{Br}$ & $44.3(56)$ & $35^{\mathrm{a}}$ & 12.4 \\
\hline
\end{tabular}

(b)

\begin{tabular}{ccrrrr}
\hline $\mathbf{R}$ & $\begin{array}{c}\mathbf{S n C l}_{\mathbf{4}} \\
{[\mathbf{g}(\mathbf{m m o l})]}\end{array}$ & $\begin{array}{c}\mathbf{R}_{\mathbf{4}} \mathbf{S n} \\
{[\mathbf{g}(\mathbf{m m o l})]}\end{array}$ & $\begin{array}{c}\mathbf{R}_{\mathbf{2}} \mathbf{S n C l}_{\mathbf{2}} \\
{[\mathbf{g}(\mathbf{m m o l})]}\end{array}$ & $\begin{array}{r}\text { Yield } \\
{[\%]}\end{array}$ & $\begin{array}{c}\boldsymbol{T}_{\mathbf{m}} \\
{\left[{ }^{\circ} \mathbf{C}\right]}\end{array}$ \\
\hline $\mathbf{P r}$ & $10.81(37)$ & $9.64(37)$ & $11.42(21)$ & 56 & 81 \\
$\mathbf{P e}$ & $9.73(37)$ & $15.06(37)$ & $20.12(61)$ & 81 & 37 \\
$\mathbf{H e x}$ & $2.83(11)$ & $5.00(11)$ & $6.54(18)$ & 83 & 46 \\
Dod & $14.00(54)$ & $42.70(54)$ & $32.90(63)$ & 58 & 52 \\
\hline
\end{tabular}

(c)

\begin{tabular}{|c|c|c|c|c|c|c|c|}
\hline $\mathbf{R}$ & $\begin{array}{c}\mathbf{R}_{2} \mathbf{S n C l}_{2} \\
{[\mathrm{~g}(\mathbf{m m o l})]}\end{array}$ & $\begin{array}{c}\mathrm{LiAlH}_{4} \\
{[\mathrm{~g}(\mathrm{mmol})]}\end{array}$ & $\begin{array}{c}\mathbf{R}_{2} \mathrm{SnH}_{2} \\
{[\mathrm{~g}(\mathrm{mmol})]}\end{array}$ & $\begin{array}{c}\text { Yield } \\
{[\%]}\end{array}$ & $\begin{array}{c}T_{\mathbf{m}} \\
{\left[{ }^{\circ} \mathbf{C}\right]}\end{array}$ & $\begin{array}{c}T_{\mathrm{b}}\left[{ }^{\circ} \mathbf{C}\right] \\
\left(\mathbf{p}_{\mathrm{b}}[\mathrm{mbar}]\right)\end{array}$ & $\begin{array}{c}\mathrm{v}(\mathbf{S n}-\mathbf{H}) \\
{\left[\mathrm{cm}^{-1}\right]}\end{array}$ \\
\hline Et & $10.00(40)$ & $2.25(59)$ & $2.00(11)$ & $28^{1}$ & & $30(81)$ & 1835 \\
\hline Pr & $6.00(22)$ & $1.25(33)$ & $2.15(11)$ & $48^{1}$ & & $24(18)$ & 1834 \\
\hline Bu & $40.00(130)$ & $8.00(210)$ & $26.57(113)$ & $87^{1}$ & & $27(1.7)$ & 1833 \\
\hline Pe & $13.00(39)$ & $2.20(58)$ & $6.72(26)$ & $65^{1}$ & & $58(0.5)$ & 1833 \\
\hline Hex & $6.22(17)$ & $1.00(26)$ & $4.47(15)$ & $89^{2}$ & -41 & & 1833 \\
\hline Oc & $26.00(63)$ & $3.50(92)$ & $20.16(58)$ & $93^{2}$ & -10.5 & & 1834 \\
\hline Dod & $10.00(19)$ & 0.72 (19) & $8.12(18)$ & $93^{2}$ & 20.2 & & 1833 \\
\hline
\end{tabular}


Table 2: ${ }^{13} \mathrm{C}$ and ${ }^{119} \mathrm{Sn}$ NMR data for the synthesized tin precursors and monomers.

Chemical shifts $(\delta)$ are given in ppm and coupling constants $(J)$ in $\mathrm{Hz}$.

\begin{tabular}{|c|c|c|c|c|c|c|c|}
\hline \multirow{2}{*}{ Compound } & \multicolumn{2}{|c|}{$-\mathrm{CH}_{3}$} & \multicolumn{2}{|c|}{$-\mathrm{CH}_{2-}$} & \multicolumn{2}{|c|}{$\mathrm{Sn}-\mathrm{CH}_{2}$} & \multirow{2}{*}{$\frac{\mathrm{Sn}}{\delta^{119} \mathrm{Sr}}$} \\
\hline & $\delta^{13} \mathrm{C}$ & $J(\mathrm{Sn}, \mathrm{C})$ & $\delta^{13} \mathrm{C}$ & $J(\mathrm{Sn}, \mathrm{C})$ & $\delta^{13} \mathrm{C}$ & $J\left({ }^{117 / 119} \mathrm{Sn}, \mathrm{C}\right)$ & \\
\hline $\mathrm{Pr}_{4} \mathrm{Sn}^{3}$ & 19.24 & ${ }^{3} J 52$ & $\begin{array}{l}20.92 \\
22.71\end{array}$ & ${ }^{2} J 20$ & 12.13 & ${ }^{i} J 299 / 312$ & -17.63 \\
\hline $\mathrm{Pe}_{4} \mathrm{Sn}^{3}$ & 14.30 & & $\begin{array}{l}27.18 \\
37.15 \\
23.12\end{array}$ & $\begin{array}{l}2 J 20 \\
3^{3} J 49\end{array}$ & 9.39 & ${ }^{2} J 298 / 312$ & -12.91 \\
\hline $\mathrm{Hex}_{4} \mathrm{Sn}^{\mathrm{a}}$ & 14.34 & & $\begin{array}{l}27.50 \\
31.92 \\
34.65 \\
23.07 \\
27.53\end{array}$ & $\begin{array}{l}{ }^{2} J 20 \\
{ }^{3} J 49 \\
{ }^{2} J 20\end{array}$ & 9.45 & ${ }^{2} J 298 / 311$ & -12.96 \\
\hline $\operatorname{Dod}_{4} \mathrm{Sn}^{2}$ & 14.21 & & $\begin{array}{l}29.80^{\mathrm{i}} \\
30.18^{\mathrm{i}} \\
32.38 \\
34.95\end{array}$ & ${ }^{3} \sqrt{50}$ & 9.74 & ${ }^{I} J 298 / 311$ & -12.93 \\
\hline $\mathrm{Pr}_{2} \mathrm{SnCl}_{2}{ }^{a}$ & 17.51 & & $\begin{array}{l}28.15 \\
2438\end{array}$ & & 18.70 & & 124.81 \\
\hline $\mathrm{Pe}_{2} \mathrm{SnCl}_{2}{ }^{\mathrm{a}}$ & 13.63 & & $\begin{array}{l}24.38 \\
25.76 \\
35.13 \\
25.03\end{array}$ & & 21.99 & & 122.85 \\
\hline $\mathrm{Hex}_{2} \mathrm{SnCl}_{2}{ }^{\mathrm{a}}$ & 14.34 & & $\begin{array}{l}26.01 \\
31.48 \\
33.11 \\
25.14 \\
26.13 \\
29.44 \\
29.90\end{array}$ & & 22.84 & & 124.99 \\
\hline $\operatorname{Dod}_{2} \mathrm{SnCl}_{2}{ }^{\mathrm{a}}$ & 14.36 & & $\begin{array}{l}29.94 \\
30.13 \\
30.18 \\
30.19 \\
32.41 \\
33.50\end{array}$ & & 23.16 & & 124.58 \\
\hline $\begin{array}{l}\mathrm{Et}_{2} \mathrm{SnH}_{2}{ }^{a} \\
\mathrm{Pr}_{2} \mathrm{SnH}_{2}{ }^{a}\end{array}$ & $\begin{array}{l}12.61 \\
18.41\end{array}$ & $\begin{array}{l}2.726 \\
3 \\
356\end{array}$ & 21.89 & ${ }^{2} J 24$ & $\begin{array}{r}-1.21 \\
9.82\end{array}$ & $\begin{array}{l}{ }^{1} J 362 / 379 \\
{ }^{2} J 358 / 375\end{array}$ & $\begin{array}{l}-181.82 \\
-208.44\end{array}$ \\
\hline $\mathrm{Bu}_{2} \mathrm{SnH}_{2}{ }^{\mathrm{b}}$ & 14.24 & & $\begin{array}{l}27.59 \\
31.11 \\
22.24\end{array}$ & ${ }^{2} J 23$ & 7.51 & ${ }^{1} J 358 / 375$ & -203.93 \\
\hline $\mathrm{Pe}_{2} \mathrm{SnH}_{2}{ }^{\mathrm{a}}$ & 13.85 & & $\begin{array}{l}27.86 \\
36.07 \\
23.01\end{array}$ & $\begin{array}{l}{ }^{2} J 24 \\
{ }^{3} J 57\end{array}$ & 6.95 & ${ }^{2} J 358 / 374$ & -203.57 \\
\hline $\operatorname{Hex}_{2} \mathrm{SnH}_{2}{ }^{a}$ & 14.31 & & $\begin{array}{l}28.52 \\
31.80 \\
33.95\end{array}$ & $\begin{array}{c}{ }^{2} J 24 \\
{ }^{3} J 54 / 57\end{array}$ & 7.38 & ${ }^{1} J 357 / 374$ & -203.63 \\
\hline $\mathrm{Oc}_{2} \mathrm{SnH}_{2}{ }^{\mathrm{a}}$ & 14.36 & & $\begin{array}{l}23.14 \\
28.62 \\
29.65 \\
29.78 \\
32.40 \\
34.34\end{array}$ & ${ }^{2} J 23$ & 7.43 & ${ }^{I} J 357 / 374$ & -203.76 \\
\hline $\operatorname{Dod}_{2} \mathrm{SnH}_{2}{ }^{\mathrm{a}}$ & 14.36 & & $\begin{array}{c}34.34 \\
23.11 \\
28.61 \\
29.71 \\
29.91 \\
30.14 \\
30.20 \\
30.24^{\mathrm{i}} \\
32.42 \\
34.34\end{array}$ & ${ }^{2} J 24$ & 7.43 & ${ }^{l} J 355 / 374$ & -203.91 \\
\hline
\end{tabular}

\footnotetext{
${ }^{\mathrm{i}}$ broad, overlapping signals; measured in ${ }^{\mathrm{a}}$ toluene- $-\mathrm{d}^{8} ;{ }^{\mathrm{b}}$ benzene- $\mathrm{d}^{6}$
} 
Table 3: ${ }^{1} \mathrm{H}$ NMR data for the synthesized tin precursors and monomers. Chemical shifts $(\delta)$ are given in ppm and coupling constants $(J)$ in $\mathrm{Hz} ; \mathrm{t}=$ triplet, $\mathrm{q}=$ quintet, se $=$ septet, $\mathrm{m}=$ multiplet.

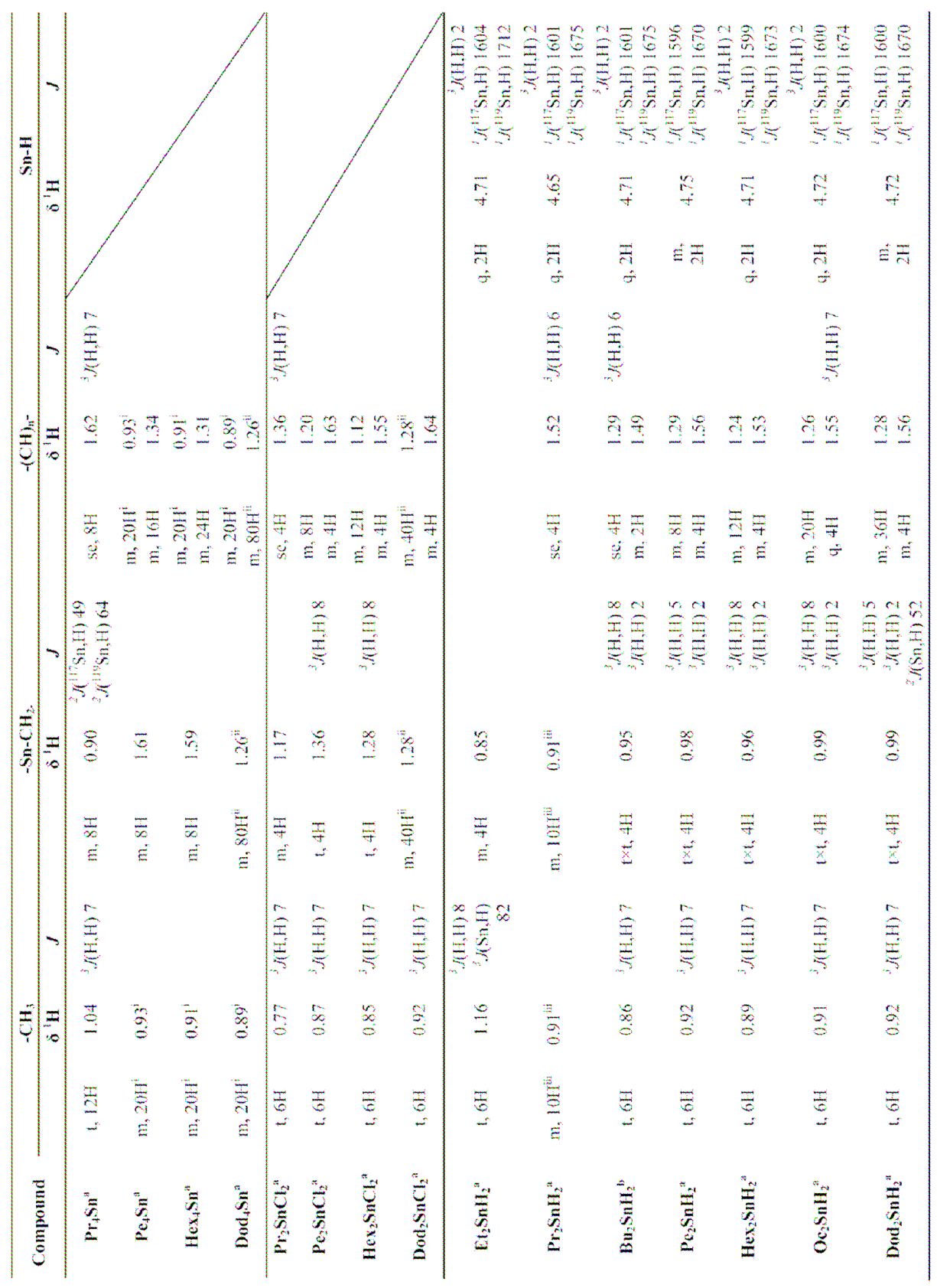

Measured in ${ }^{a}$ toluene- $d^{8} ;{ }^{b}$ benzene- $d^{6} ;{ }^{i}$ overlapping of signals from the $-\mathrm{CH}_{3}$ and $-\mathrm{CH}_{2}-$ groups; ${ }^{\text {ii }}$ overlapping of the signals of $-\mathrm{CH}_{2}$ - and $\mathrm{Sn}-\mathrm{CH}_{2}$ - groups; $;{ }^{\text {iii }}$ overlapping of the signals of a $-\mathrm{CH}_{3}$ and $\mathrm{Sn}-\mathrm{CH}_{2}$ - group 
Table 4: Calculated and found chemical composition (in $\% \mathrm{~m} / \mathrm{m}$ ) of dialkyldichlorostannes and dialkylstannanes.

\begin{tabular}{|c|c|c|c|c|c|}
\hline Compound & & $\mathbf{C}$ & $\mathbf{H}$ & $\mathbf{C l}$ & Purification \\
\hline \multirow{2}{*}{$\mathrm{Pr}_{2} \mathrm{SnCl}_{2}$} & found & 26.08 & 4.95 & 25.79 & recrystallization \\
\hline & calc. & 26.13 & 5.12 & 25.71 & (pentane at $-78^{\circ} \mathrm{C}$ ) \\
\hline \multirow[b]{2}{*}{$\mathrm{Pe}_{2} \mathrm{SnCl}_{2}$} & found & 35.89 & 6.59 & 21.47 & recrystallization \\
\hline & calc & 36.19 & 6.68 & 21.36 & (pentane at $-78^{\circ} \mathrm{C}$ ) \\
\hline \multirow{2}{*}{$\mathrm{Hex}_{2} \mathrm{SnCl}_{2}$} & found & 40.19 & 7.20 & 19.84 & recrystallization \\
\hline & calc. & 40.04 & 7.28 & 19.70 & (pentane at $-78^{\circ} \mathrm{C}$ ) \\
\hline \multirow{2}{*}{$\operatorname{Dod}_{2} \mathrm{SnCl}_{2}$} & found & 54.84 & 9.81 & 13.45 & recrystallization \\
\hline & calc. & 54.57 & 9.54 & 13.42 & (pentane at $-78^{\circ} \mathrm{C}$ ) \\
\hline \multirow{2}{*}{$\mathbf{E t}_{2} \mathrm{SnH}_{2}$} & found & 27.33 & 6.34 & n.a. & distillation \\
\hline & calc. & 26.86 & 6.76 & n.a. & $\left(81 \mathrm{mbar}\right.$ at $\left.30^{\circ} \mathrm{C}\right)$ \\
\hline \multirow{2}{*}{$\mathrm{Pr}_{2} \mathrm{SnH}_{2}$} & found & 35.11 & 7.82 & n.a. & distillation \\
\hline & calc. & 34.83 & 7.79 & n.a. & $\left(18\right.$ mbar at $\left.24^{\circ} \mathrm{C}\right)$ \\
\hline \multirow{2}{*}{$\mathrm{Bu}_{2} \mathrm{SnH}_{2}$} & found & 40.84 & 8.47 & n.a. & distillation \\
\hline & calc. & 40.90 & 8.58 & n.a. & $\left(1.7 \mathrm{mbar}\right.$ at $\left.26^{\circ} \mathrm{C}\right)$ \\
\hline \multirow{2}{*}{$\mathrm{Pe}_{2} \mathrm{SnH}_{2}$} & found & 45.37 & 9.27 & n.a. & distillation \\
\hline & calc & 45.67 & 9.20 & n.a. & $\left(0.5\right.$ mbar at $\left.58^{\circ} \mathrm{C}\right)$ \\
\hline \multirow{2}{*}{$\mathrm{Hex}_{2} \mathrm{SnH}_{2}$} & found & 49.59 & 9.70 & n.a. & recrystallization \\
\hline & calc. & 49.52 & 9.70 & n.a. & (pentane at $-78^{\circ} \mathrm{C}$ ) \\
\hline \multirow{2}{*}{$\mathrm{Oc}_{2} \mathrm{SnH}_{2}$} & found & 55.83 & 9.97 & n.a. & recrystallization \\
\hline & calc. & 55.35 & 10.45 & n.a. & (pentane at $-78^{\circ} \mathrm{C}$ ) \\
\hline \multirow{2}{*}{$\operatorname{Dod}_{2} \mathrm{SnH}_{2}$} & found & 63.34 & 11.36 & n.a. & recrystallization \\
\hline & calc. & 62.75 & 11.41 & n.a. & (ether at $-30{ }^{\circ} \mathrm{C}$ ) \\
\hline
\end{tabular}

n.a.: not applicable 


\section{References}

(1) Grignard, V. C. R. Hebd. Séances Acad. Sci. 1900, 130, 1322-1324.

(2) Imori, T.; Lu, V.; Cai, H.; Tilley, T. D. J. Am. Chem. Soc. 1995, 117, 9931-9940.

(3) Bokranz, A.; Plum, H. Fortschr. Chem. Forsch. 1971, 16, 365-403.

(4) van der Kerk, G. J. M.; Luijten, J. G. A. J. Appl. Chem. 1957, 7, 369-374.

(5) Kozeschkow, K. A. Ber. Deutsch. Chem. Gesell. 1933, 66, 1661-1665.

(6) Choffat, F.; Smith, P.; Caseri, W. J. Mater. Chem. 2005, 15, 1789-1792.

(7) Finholt, A. E.; Bond, A. C.; Schlesinger, H. I. J. Am. Chem. Soc. 1947, 69, 1199-1203.

(8) Finholt, A. E.; Bond, A. C.; Wilzbach, K. E.; Schlesinger, H. I. J. Am. Chem. Soc. 1947, 69, 2692-2696. 